\title{
Neurobiological research on addiction: What value has it added to the concept?
}

\author{
Harold Kalant \\ Department of Pharmacology \& Toxicology, University of Toronto and Centre for Addiction and Mental Health, Toronto, Canada
}

\begin{abstract}
The initial goal of neurobiological research on addiction was to identify the neural mechanisms involved in the mediation and expression of addictive behavior. More recently, however, it has attributed causal roles to these mechanisms, as illustrated by the definition of addiction as a brain disease caused by chronic exposure to a drug. This concept carries a number of implications that can be assessed experimentally and clinically. None of these implications is borne out by the currently available evidence. The interactions of neuronal systems involved in addiction are also involved in adaptation to experience and environmental change. Much of the neurobiological research to date has not differentiated between causes of addiction, neuronal mechanisms that are activated by them, and risk factors that contribute to individual vulnerability. It has largely ignored the important experiential and environmental influences known to affect the prevalence of addiction in different populations or different times, and it has so far directed much less attention to other forms of addiction-like behavior that do not involve drugs. These failures are not inherent in neurobiological research but require reorientation of objectives, including more emphasis on the study of mechanisms by which environment and experience, including drug experience, can determine whether genetic risk factors are expressed or remain dormant and can direct neuroadaptive mechanisms toward alternative outcomes.
\end{abstract}

\section{Changing Concepts of Addiction}

During the 19th century and the first half of the 20th century, the term drug addiction gradually evolved as a medical replacement for the earlier concept of excessive use of alcohol, opium, and other drugs as a problem of moral weakness. It placed increasing emphasis on the biochemical actions of the drugs as the causal factor (Berridge, 1990), as represented by a World Health Organization (WHO) definition of addiction as "a state of periodic or chronic intoxication, detrimental to the individual and to society, produced by the repeated consumption of a drug." In the second half of the century the term addiction was replaced by dependence in most of the official systems of nomenclature of disease; it was defined in terms of the behavior of the user, the essential features being a persistent excessive use of the drug despite adverse consequences and great difficulty in ceasing such use, as shown by the high frequency of relapse (American Psychological Association, 1994). Nevertheless, the term addiction continued in wide use as the fully interchangeable equivalent of dependence. The American Psychological Association (APA) definition was quite consistent with the original meaning of addiction in ancient Rome, which was the state of a debtor legally bound over in slavery to a creditor until the debt was paid. The essential concept, both in ancient Rome and in the late 20th-century meaning of addiction, was a loss of freedom of action by the affected individual.
In the past two decades, neurobiological research has made brilliant advances in the understanding of the functional organization and mechanisms of the brain, and much of this work has been directed at brain alterations accompanying the development of addiction. This has led to a neurobiological definition of addiction as a chronic, relapsing brain disorder that results from the prolonged effects of drugs on the brain and is characterized by compulsive drug-seeking and use, despite harmful consequences (Leshner, 1997). This is in some ways a regression to the earlier concept embodied in the WHO definition cited above, since it states clearly that the actions of the drug cause the brain changes that are presumed to be the essence of addiction.

At the risk of oversimplification, one may reduce this neurobiological concept of addiction to the following steps. People use drugs because the drugs act upon a postulated "reward system" in the brain, and the users thus receive some benefit or pleasure (the "reward") from them, at least initially. However, even a single use of the drug gives rise to "opponent processes" (Koob, 2009) that are opposite in direction to the effect of the drug and thus produce a progressive decrease in drug effect, recognized as acute tolerance. After elimination of the drug the opponent processes are manifested as an acute withdrawal effect (LeBlanc, Kalant, \& Gibbins, 1975; McQuarrie \& Fingl, 1958). Repeated heavy use of the drug leads to longer-

Correspondence: Prof. H. Kalant, Department of Pharmacology \& Toxicology, Medical Sciences Building, Room 4207, 1 King’s College Circle, University of Toronto, Toronto, Canada M5S 1A8. E-mail address: harold.kalant@utoronto.ca

Keywords: Addiction; Neuronal adaptation; Environmental influences; Genetic factors; Epigenetics 
lasting adaptive changes that constitute the physiological basis of physical dependence but also removes the reward system from its normal controls, replacing normal homeostasis with an altered state ("allostasis") such that drug consumption does not lead to satiation but to "compulsive" use of ever more drug. The nature of this altered state is not yet known, but it constitutes the essence of addiction, according to this conceptual model.

One of the problems in evaluating this concept of addiction is that most of the neurobiological research on brain changes presumed to underlie addiction has not employed behavioral tests that truly demonstrate the presence of addiction in the experimental subjects. In an excellent and comprehensive review, Winger, Woods, Galuska, and Wade-Galuska (2005) have pointed out that the tests most commonly used, such as drug-conditioned place preference or sensitization of drug-induced locomotor activity, reflect motivational properties of the drugs but do not provide evidence of the so-called compulsive drug use and great difficulty of cessation of such use that are the defining features of addiction.

\section{Dopamine and the Reward System}

Obviously the brain is involved in addiction, as it is in all behaviors, but several different types of evidence argue strongly against the concept that drug action on the brain reward system is per se the cause of addiction. One problem is that the reward system itself is not yet satisfactorily defined. Several types of evidence argue against the simplistic view that dopaminergic projections from the ventral tegmental area (VTA) of the midbrain to the nucleus accumbens and the prefrontal cortex (the mesolimbic dopamine pathways) constitute the reward system (Kalant, 2010; Winger et al., 2005). Though the mesolimbic dopamine system is preferentially activated by stimuli predictive of presentation of a reward, under some conditions it can also be activated to a lesser extent by stimuli predicting punishment or by a motivationally neutral but unusually intense stimulus (Mirenowicz \& Schultz, 1996). Thus, an alternative view of the role of this dopamine pathway is that it alerts the brain to environmental changes that may be important for the organism, whether as rewards, as punishments, or as signals of potential threats. This view receives some support from the observation that different types of response of the mesolimbic dopamine system are elicited by different types of stimulus (Schultz, 2007). Therefore, the fact that all drugs of dependence can stimulate the mesolimbic dopamine system does not prove that this action is the direct cause of addiction or even that the activated dopamine pathways are the reward system.

An alternative explanation is that the dopamine-activated projections from the nucleus accumbens to the prefrontal cortex are part of a brain system mediating executive functions, such as problem-solving, working memory, and comparative assessment and rational weighing of future outcomes. These functions are proposed as the basis of associative learning, based on the comparison of predicted reward and actual reward (Everitt et al., 1999; Rogers et al., 1999). On this basis, addiction is seen as the result of dominance of an immediate response system, based on the dopaminergic pathways from the VTA to the accumbens, over a system mediating delayed but rationally assessed response, based on the prefrontal cortex (Bechara, 2005; Bickel et al., 2007; Olausson et al., 2007).

\section{Implications of a Purely Neurobiological Concept of Addiction}

The exclusively neurobiological concept of addiction carries a number of implications. If the drug action alone is the cause, there should be something distinctive about drug activation of the reward system that differentiates it from activation of that system by non-drug reinforcers. Repeated activation by drugs should invariably give rise to addiction. It should not matter whether the exposed individual has actively self-administered the drug or has been passively exposed to it. Behavioral and environmental influences should have little or no influence on the development of addiction. Genetic factors should be important because some of them enhance or interfere with the action of the drug. Other behaviors that are commonly termed addictions, such as compulsive overeating or compulsive gambling, that do not involve drug use should have different cellular mechanisms than those of drug addiction. If one can find the exact locus of the brain changes and their molecular mechanisms, it should be possible to find a medication to reverse the changes and thus eliminate the addiction. All of these implications are experimentally testable, and therefore it should be possible to determine whether that concept of addiction is supported by the evidence.

\section{Evidence That Does Not Support an Exclusively Neurobiological Concept}

The first implication is contradicted by electrophysiological observations of neuronal responses in the brains of monkeys trained to perform simple tests in order to obtain cocaine, water, or fruit juice when the respective availabilities were signaled by differential signals (Deadwyler, 2010; Deadwyler, Hayashizaki, Cheer, \& Hampson, 2004; Opris, Hampson, \& Deadwyler, 2009). Three different populations of dopamine-activated neurons in the nucleus accumbens were observed: those activated only by presentation of the signal for the natural reinforcer (juice or water), those activated only by the signal for cocaine, and a majority activated by both, but in all cases the pattern of activation was essentially the same. The specificity of behavioral responses appeared to result from the differing signals and contexts that predicted the different rewards and that activated different downstream neuronal networks through associative learning. The activation of the different nucleus accumbens neurons themselves by the different rewards appeared to differ only as a result of the reward value or strength, or the salience of the different rewards, in much the same way as the differences found in response to different concentrations of sugar solution or to different volumes of water (e.g., Tobler, Fiorillo, \& Schultz, 2005).

The second implication is clearly not correct. Even though different drugs carry different degrees of risk, it is 
obvious that the majority of users of alcohol or other psychoactive drugs do not become addicted, despite long periods of repeated use.

The third implication is possibly refuted by the finding that it does matter whether the exposed individual has or has not self-administered the drug. The pattern of regional metabolic activation of the rat brain after rewarding electrical self-stimulation of the VTA or cocaine is quite different from the pattern seen after stimulation of the same area by the experimenter (Howell, Votaw, Goodman, \& Lindsey, 2010; Porrino et al., 1984). It is widely recognized that hospitalized patients to whom high doses of opioid drugs are administered by treatment personnel for relief of severe pain frequently develop tolerance and physical dependence, yet they seldom become compulsive drug seekers and users (O'Brien, 2001). In contrast, patients who are allowed to self-administer the drug do frequently become addicted, as shown by recent experience with high-dose oral oxycodone in the United States and Canada (Brands, Blake, Sproule, Gourlay, \& Busto, 2004; Hays, 2004; Knudsen, Ducharme, \& Roman, 2007). Thus, it appears that the experience of drug effect as a consequence of the user's own behavior is related to the production of addiction. However, caution is necessary in drawing a conclusion on this point. Patients with severe pain are more likely to be receiving opiates administered by health care personnel or other persons rather than by themselves. Severe pain can modify the response to exogenous opioids by altering endogenous opioid activity; for example, in some rat models resistance to neuropathic pain, or recovery from it, is associated with increased activity of endogenous opioid systems (Herradon et al., 2008; Obara, Mika, Schafer, \& Przewlocka, 2003; Stagg et al., 2011). Therefore, one cannot exclude the possibility that severe pain modifies the drug response in some manner that reduces the risk of addiction.

The fourth implication of the neurobiological concept of addiction is also contradicted by experimental evidence that environmental factors can have a substantial influence on drug-taking (Badiani \& Robinson, 2004). Rats that were given the opportunity to self-administer cocaine by intravenous injection acquired the self-administration behavior more quickly and used larger amounts if the drugtaking occurred in a novel environment than in the familiar home environment, and the animals also reached a higher break point on a progressive ratio schedule of reinforcement (i.e., they worked harder to get the drug) in the novel environment than in the home environment (Caprioli et al., 2007). Similarly, rats acquired selfadministration of ketamine and of amphetamine more rapidly and took much larger amounts when drug access occurred in a novel environment than in the home environment (Caprioli et al., 2008; De Luca \& Badiani, 2011). In contrast, rats acquired self-administration of heroin and of alcohol more rapidly in the home environment than in a novel environment (Caprioli et al., 2009; Testa, Nencini, \& Badiani, 2011), but the relevant point in the present context is the important role played by the environment in determining the preference for these drugs also.
A striking example in humans is provided by the difference in relapse risk in treated heroin addicts in two different studies. American soldiers who had become addicted to heroin during their participation in the Vietnam war, who were treated on their return to the United States and then were discharged to their family homes and surroundings, had very low rates of relapse (Robins, Helzer, \& Davis, 1975). In contrast, American heroin addicts who had been treated for two years or more in a U.S. Public Health Service Hospital and were free of craving and protracted withdrawal symptoms at the time of their discharge relapsed rapidly on their return to the city environments associated with their previous drug use (Wikler, 1959).

One of the important environmental influences on risk of addiction is peer-group influence. This has long been recognized in humans but has recently been shown in laboratory animal studies, in which speech, persuasion, and provision of drug cannot play a role. Peer influence between prairie voles housed in adjoining cage segments can either increase or decrease ethanol consumption, depending upon the experimental conditions (Anacker, Loftis, Kaur, \& Ryabinin, 2011; Anacker, Loftis, \& Ryabinin, 2011). Individually and separately housed voles were given a free choice of water and $10 \%$ ethanol for a period of days and were classified as either high or low drinkers. High drinkers and low drinkers were then paired in conjoined cages separated by a wire mesh partition, and again each animal was given a choice of water and ethanol. About half of the high drinkers decreased their intake to that of the paired low drinkers, while the other half did not (Anacker \& Ryabinin, 2013).

The fifth implication, concerning the importance of genetic factors, is indeed true, but not to the extent emphasized in the neurobiological literature. It is now recognized that there is no single gene for addiction or for any individual form of addiction; rather, a very large number of genes make individually small contributions to susceptibility to addiction (Li, Mao, \& Wei, 2008; Mayfield, Harris, \& Schuckit, 2008; Schuckit et al., 2001; Winger et al., 2005). The large number of genes involved encode proteins required in virtually every type of neuronal activity, including synthesis, storage, release and degradation of neurotransmitters, receptors, ion channels, intracellular signaling pathways, nuclear transcription factors, and others. The sum of these many small contributions results, in the case of alcoholism and other drug addictions, in a heritability of roughly $50 \%$, so that the other half of the explanation of the causation requires the involvement of behavioral and environmental factors of diverse kinds (Mayfield et al., 2008; Winger et al., 2005).

A more recent approach to the genetics of addiction, therefore, has been to study the genetics of behavioral traits that may represent predisposing factors. For example, impulsivity in rats, as measured by inability to postpone a response to a reward signal when a brief postponement would yield a much greater reward, is correlated with more rapid acquisition of cocaine self-administration, even when the latter is accompanied by an aversive footshock (Belin, Mar, Dalley, Robbins, \& Everitt, 2008; Dalley et al., 2007). 
It is also correlated with higher consumption of ethanol and greater probability of relapse after extinction of the drinking behavior (Poulos, Parker, \& Lê, 1996). However, the correlation of degree of impulsivity with amount of cocaine self-administration, though statistically significant, is not very precise (Belin et al., 2008), and impulsivity is correlated with other behavioral disorders unrelated to the use of drugs, such as gambling (el-Guebaly, Mudry, Zohar, Tavares, \& Potenza, 2012; Odlaug, Schreiber, \& Grant, 2013) and compulsive buying (Yi, 2013). Therefore, impulsivity must be considered a contributory risk factor for addiction but not a direct cause.

The sixth implication of the neurobiological concept of addiction mentioned above is inconsistent with the relatively small amount of experimental and clinical evidence available on so-called behavioral addictions such as pathological gambling and pathological overeating. Various types of evidence suggest that these behavioral alterations are brought about by closely similar, or perhaps the same, mechanisms as those operating in the development of drug addictions (Blum, Liu, Shriner, \& Gold, 2011; Kelley, Schiltz, \& Landry, 2005). For example, rats can be trained to "binge" on chow or sugar solution by limiting the amount of time each day in which they have access to it. The time-restricted animals take in much more food than those that have unlimited access, and they develop concurrent brain changes closely resembling those found in drug-dependent animals, including naloxone-precipitable withdrawal signs (Avena, Rada, \& Hoebel, 2008; Hagan \& Moss, 1997; Hoebel, Avena, Bocarsly, \& Rada, 2009; Rada, Avena, \& Hoebel, 2005). The stimulus strength of very sweet solutions, as reflected in the preference for either saccharine or sugar solution, exceeds that of cocaine solution, even in cocaine-dependent animals (Lenoir, Serre, Cantin, \& Ahmed, 2007). In humans, binging on sweet palatable foods activates dopamine release in the nucleus accumbens and is positively reinforcing. Chronic binging is accompanied by changes in the release of dopamine, acetylcholine, and enkephalin in the nucleus accumbens, and cessation of this behavior is followed by anxiety, depression, and craving for the absent reward (Bello \& Hajnal, 2010; Colantuoni et al., 2002). There are also reports that naltrexone has been effective in aiding termination of this form of excessive eating, and naloxone was reported to be as effective in reducing excessive intake of sugar and saccharine solutions as of ethanol by the rat (Cichelli \& Lewis, 2002).

The seventh implication mentioned above is also at odds with the relatively limited value of drug therapies for addiction that have been developed so far. Perhaps the most attention has been directed to the use of naltrexone and nalmefene in the treatment of alcoholism (Hillemacher, Heberlein, Muschler, Bleich, \& Frieling, 2011; Rosner et al., 2010), opioid addiction, compulsive overeating, and other addictions. It has certainly helped maintain abstinence during the early weeks of treatment, thus giving sociobehavioral therapy a greater opportunity to work, but with the passage of time the beneficial effect progressively lessens (Anton et al., 2006; Mason, 2003; Yahn, Watterson, \& Olive, 2013), even during continuous treatment for up to
15 months with injectable slow-release preparations (Bart, 2012; Lee et al., 2012). Whether such preparations will be useful in maintaining long-term reduction of alcohol intake as opposed to total abstinence remains to be determined.

\section{Mechanism Versus Cause}

In addition to the evidence that is not consistent with the implications of the neurobiological explanation of drug addiction as described above, there is a further argument against it that is based on its failure to distinguish between a mechanism and a cause. As pointed out in detail elsewhere (Kalant, 2010; Robbins \& Everitt, 1999; Winger et al., 2005), all of the neuronal mechanisms and pathways that have been implicated in addiction have also been shown to be involved in learning, memory, and other forms of neuroadaptation. Therefore, they can be regarded as the basic machinery of adaptation, but the term cause must be reserved for that which sets the machinery in motion and directs it toward a particular target or outcome.

\section{Brain-Environment Interactions as the Proper Target of Neurobiological Study of Addiction}

In the case of drug addiction, a variety of social and environmental factors, as well as genetic influences on susceptibility, have been demonstrated. Among these are the cost of alcohol and other drugs relative to disposable income, the ease of accessibility of the substances used, social traditions and attitudes toward their use, expectations concerning their effects (Stein, Goldman, \& Del Boca, 2000), family and peer-group examples and pressures, and even the physical environment in which drug use occurs. The models proposed by Bechara (2005), Bickel et al. (2007), and Olausson et al. (2007), cited above, clearly implicate such factors as essential components of the brainenvironment interaction that they consider to be the cause of addiction. However, how these factors interact with the brain mechanisms to set in motion the behaviors and reactions leading to addiction has so far received relatively little scientific attention. One possible mechanism might involve sensory inputs from the environment acting to modulate the expression of genes that contribute to susceptibility or resistance to addiction. Another might be direct action of such inputs on impulse transmission at synapses in the pathways between the midbrain and the prefrontal cortex. These are indeed questions which neurobiological research is wonderfully well equipped to tackle. Therefore, though it has so far added relatively little to the concept of addiction, if it devotes more of its research effort towards brain-environment interactions, it offers future possibilities of much greater added value not only for conceptual advances but also for practical gains in prevention and treatment.

\section{References}

American Psychiatric Association. (1994). Diagnostic and statistical manual of mental disorders (4 $4^{\text {th }}$ ed.). Washington, DC, United States: Author.

Anacker, A. M., Loftis, J. M., Kaur, S., \& Ryabinin, A. E. (2011). Prairie voles as a novel model of socially 
facilitated excessive drinking. Addiction Biology, 16, 92-107.

Anacker, A. M., Loftis, J. M., \& Ryabinin, A. E. (2011). Alcohol intake in prairie voles is influenced by the drinking level of a peer. Alcoholism: Clinical and Experimental Research, 35, 1884-1890.

Anacker, A. M. J., \& Ryabinin, A. E. (2013). Identification of subpopulations of prairie voles differentially susceptible to peer influence to decrease high alcohol intake. Frontiers in Pharmacology 4, 84. doi:10.3389/fphar.2013.00084

Anton, R. F, O'Malley, S. S., Ciraulo, D. A., Cisler, R. A., Couper, D., Donovan, D. M., .. COMBINE Study Research Group. (2006). Combined pharmacotherapies and behavioral interventions for alcohol dependence. The COMBINE study: A randomized clinical trial. JAMA, 295, 2003-2017.

Avena, N. M., Rada, P., \& Hoebel, B. G. (2008). Evidence for sugar addiction: Behavioral and neurochemical effects of intermittent, excessive sugar intake. Neuroscience \& Biobehavioral Reviews, 32, 20-39.

Badiani, A., \& Robinson, T. E. (2004). Drug-induced neurobehavioral plasticity: The role of environmental context. Behavioral Pharmacology, 15, 327-339.

Bart, G. (2012). Maintenance medication for opiate addiction: The foundation of recovery. Journal of Addictive Diseases, 31, 207-225.

Bechara, A. (2005). Decision making, impulse control, and loss of willpower to resist drugs: A neurocognitive perspective. Nature Neuroscience, 8, 1458-1463.

Belin, D., Mar, A. C., Dalley, J. W., Robbins, T. W., \& Everitt, B. J. (2008). High impulsivity predicts the switch to compulsive cocaine-taking. Science, 320, 1352-1355.

Bello, N. T., \& Hajnal, A. (2010). Dopamine and binge eating behaviors. Pharmacology, Biochemistry \& Behavior, 97, 25-33.

Berridge, V. (1990). Dependence: Historical concepts and constructs. In G. Edwards \& M. Lader (Eds.), The nature of drug dependence (pp. 1-18). New York, NY. United States: Oxford University Press.

Bickel, W. K., Miller, M. K., Yi, R., Kowal, B. P., Lindquist, D. M., \& Pitcock, J. A. (2007). Behavioral and neuroeconomics of drug addiction: Competing neural systems and temporal discounting processes. Drug and Alcohol Dependence, 90(Suppl.1), S85-S91.

Blum, K., Liu, Y., Shriner, R., \& Gold, M. S. (2011). Reward circuitry dopaminergic activation regulates food and drug craving behavior. Current Pharmaceutical Design, 17, 1158-1167.

Brands, B., Blake, J., Sproule, B., Gourlay, D., \& Busto, U. (2004). Prescription opioid abuse in patients presenting for methadone maintenance treatment. Drug and Alcohol Dependence, 73, 199-207.

Caprioli, D., Celentano, M., Dubla, A., Lucantonio, F., Nencini, P., \& Badiani, A. (2009). Ambience and drug choice: Cocaine- and heroin-taking as a function of environmental context in humans and rats. Biological Psychiatry, 65, 893-899.

Caprioli, D., Celentano, M., Paolone, G., Lucantonio, F., Bari, A., Nencini, P., \& Badiani, A. (2008). Opposite environmental regulation of heroin and amphetamine self-administration in the rat. Psychopharmacology, 198, 395-404.

Caprioli, D., Paolone, G., Celentano, M., Testa, A., Nencini, P., \& Badiani, A. (2007). Environmental modulation of cocaine self-administration in the rat. Psychopharmacology, 192, 397-402.

Cichelli, M. J., \& Lewis, M. J. (2002). Naloxone nonselective suppression of drinking of ethanol, sucrose, saccharine, and water by rats. Pharmacology, Biochemistry \& Behavior, 72, 699-706.

Colantuoni, C., Rada, P., McCarthy, J., Patten, C., Avena, N. M., Chadeayne, A., \& Hoebel, B. G. (2002). Evidence that intermittent, excessive sugar intake causes endogenous opioid dependence. Obesity Research, 10, 478-488.

Dalley, J. W., Fryer, T. D., Brichard, L., Robinson, E. S. J., Theobald, D. E. H., Lääne, K., ... Robbins, T. W. (2007). Nucleus accumbens D2/3 receptors predict trait impulsivity and cocaine reinforcement. Science, 315, 1267-1270.

Deadwyler, S. A. (2010). Electrophysiological correlates of abused drugs. Relation to natural rewards. Annals of the New York Academy of Sciences, 1187, 140-147.

Deadwyler, S. A., Hayashizaki, S., Cheer, J., \& Hampson, R. E. (2004). Reward, memory and substance abuse: Functional neuronal circuits in the nucleus accumbens. Neuroscience and Biobehavioral Reviews, 27, 703711.

De Luca, M. T., \& Badiani, A. (2011). Ketamine selfadministration in the rat: Evidence for a critical role of setting. Psychopharmacology, 214, 549-556.

el-Guebaly, N., Mudry, T., Zohar, J., Tavares, H., \& Potenza, M. N. (2012). Compulsive features in behavioural addictions: The case of pathologic gambling. Addiction, 107, 1735-1736.

Everitt, B. J., Parkinson, J. A., Olmstead, M. C., Arroyo, M., Robledo, P., \& Robbins, T. W. (1999). Associative processes in addiction and reward. The role of amygdala-ventral striatal subsystems. Annals of the New York Academy of Sciences, 877, 412-438.

Hagan, M. M., \& Moss, D. E. (1997). Persistence of bingeeating patterns after a history of restriction with intermittent bouts of refeeding on palatable food in rats: Implications for bulimia nervosa. International Journal of Eating Disorders, 22, 411-420.

Hays, L. R. (2004). A profile of OxyContin addiction. Journal of Addictive Diseases 23(4), 1-9.

Herradon, G., Ezquerra, L., Nguyen, T., Wang, C., Siso, A., Franklin, B., ... Alguacil, L. F. (2008). Noradrenergic and opioidergic alterations in neuropathy in different rat strains. Neuroscience Letter, 438, 186-189.

Hillemacher, T., Heberlein, A., Muschler, M. A., Bleich, S., \& Frieling, H. (2011). Opioid modulators for alcohol dependence. Expert Opinion on Investigational Drugs, 20, 1073-1086.

Hoebel, B. G., Avena, N. M., Bocarsly, M. E., \& Rada, P. (2009). Natural addiction: A behavioral and circuit model based on sugar addiction in rats. Journal of Addiction Medicine, 3, 33-41.

Howell, L. L., Votaw, J. R., Goodman, M. M., \& Lindsey, K. P. (2010). Cortical activation during cocaine use 
and extinction in rhesus monkeys. Psychopharmacology, 208, 191-199.

Kalant, H. (2010). What neurobiology cannot tell us about addiction. Addiction, 105, 780-789.

Kelley, A. E., Schiltz, C. A., \& Landry, C. F. (2005). Neural systems recruited by drug- and food-related cues: Studies of gene activation in corticolimbic regions. Physiology \& Behavior, 86, 11-14.

Knudsen, H. K., Ducharme, L. J., \& Roman, P. M. (2007). Controlled-release oxycodone admissions in public and private substance abuse treatment. Journal of Addictive Diseases, 26(1), 41-50.

Koob, G. F. (2009). Neurobiological substrates for the dark side of compulsivity in addiction. Neuropharmacology, 56(Suppl.1), 18-31.

LeBlanc, A. E., Kalant, H., \& Gibbins, R. J. (1975). Acute tolerance to ethanol in the rat. Psychopharmacologia (Berl.), 41, 43-46.

Lee, J. D., Grossman, E., Huben, L., Manseau, M., McNeely, J., Rotrosen, J., ... Gourevitch, M. N. (2012). Extended-release naltrexone plus medical management alcohol treatment in primary care: Findings at 15 months. Journal of Substance Abuse Treatment, 43, 458-462.

Lenoir, M., Serre, F., Cantin, L., \& Ahmed, S. H. (2007). Intense sweetness surpasses cocaine reward. PLoS ONE, 2(8), e698. doi:10.1371/journal.pone.0000698

Leshner, A. I. (1997). Addiction is a brain disease, and it matters. Science, 278, 45-47.

Li, C. -Y., Mao, X., \& Wei, L. (2008). Genes and (common) pathways underlying addiction. PLoS Computational Biology 4(1): e2. doi:10.1371/journal.pcbi.0040002. Retrieved from http://www.ploscompbiol.org

Mason, B. J. (2003). Acamprosate and naltrexone treatment for alcohol dependence. European Neuropsychopharmacology, 13, 469-475.

Mayfield, R. D., Harris, R. A., \& Schuckit, M. A. (2008). Genetic factors influencing alcohol dependence. British Journal of Pharmacology, 154, 275-287.

McQuarrie, D. G., \& Fingl, E. (1958). Effects of single doses and chronic administration of ethanol on experimental seizures in mice. Journal of Pharmacology and Experimental Therapeutics, 124, 264-271.

Mirenowicz, J., \& Schultz, W. (1996). Preferential activation of midbrain dopamine neurons by appetitive rather than aversive stimuli. Nature, 379, 449-451.

Obara, I., Mika, J., Schafer, M. K., \& Przewlocka, B. (2003). Antagonists of the kappa-opioid receptor enhance allodynia in rats and mice after sciatic nerve ligation. British Journal of Pharmacology, 140, 538546.

O’Brien, C. P. (2001). Drug addiction and drug abuse. In J. G. Hardman \& L. E. Limbird (Eds.), Goodman \& Gilman's the pharmacological basis of therapeutics, 10th ed. (pp. 621-644). New York, NY, United States: McGraw-Hill Medical Publishing.

Odlaug, B. L., Schreiber, L. R. N., \& Grant, J. E. (2013). Personality dimensions and disorders in pathological gambling. Current Opinion in Psychiatry, 26, 107112.
Olausson, P., Jentsch, J. D., Krueger, D. D., Tronson, N. C., Naim, A. C., \& Taylor, J. R. (2007). Orbitofrontal cortex and cognitive-motivational impairments in psychostimulant addiction: Evidence from experiments in the non-human primate. Annals of the New York Academy of Sciences, 1121, 610-638.

Opris, I., Hampson, R. E., \& Deadwyler, S. A. (2009). The encoding of cocaine vs. natural rewards in the striatum of nonhuman primates: Categories with different activations. Neuroscience, 163, 40-54.

Porrino, L. J., Esposito, R. U., Seeger, T. F., Crane, A. M., Pert, A., \& Sokoloff, L. (1984). Metabolic mapping of the brain during rewarding self-stimulation. Science, 224, 306-309.

Poulos, C. X., Parker, J. L., \& Lê, A. D. (1996). Dexfenfluramine and 8-OH-DPAT modulate impulsivity in a delay-of-reward paradigm: Implications for a correspondence with alcohol consumption. Behavioral Pharmacology, 7, 395-399.

Rada, P., Avena, N. M., \& Hoebel, B. G. (2005). Daily bingeing on sugar repeatedly releases dopamine in the accumbens shell. Neuroscience, 134, 737-744.

Robbins, T. W., \& Everitt, B. J. (1999). Drug addiction: Bad habits add up. Nature, 398, 567-570.

Robins, L. N., Helzer, J. E., \& Davis, D. H. (1975). Narcotic use in South-east Asia and afterward: An interview study of 898 Vietnam returnees. Archives of General Psychiatry, 32, 955-961.

Rogers, R. D., Owen, A. M., Middleton, H. C., Williams, E. J., Pickard, J. D., Sahakian, B. J., \& Robbins, T. W. (1999). Choosing between small, likely rewards and large, unlikely rewards activates inferior and orbital prefrontal cortex. Journal of Neuroscience, 19, 90299038.

Rosner, S., Hackl-Herrwerth, A., Leucht, S., Vecchi, S., Srisurapanont, M., \& Soyka, M. (2010). Opioid antagonists for alcohol dependence. Cochrane Database of Systematic Reviews, 12, CD001867.

Schuckit, M. A., Edenberg, H. J., Kalmijn, J., Flury, L., Smith, T. L., Reich, T., ... Foroud, T. (2001). A genome-wide search for genes that relate to a low level of response to alcohol. Alcoholism: Clinical and Experimental Research, 25, 323-329.

Schultz, W. (2007). Multiple dopamine functions at different time courses. Annual Review of Neuroscience, 30, 259-288.

Stagg, N. J., Mata, H. P., Henriksen, E. J., Porreca, F., Vanderah, T. W., \& Philip Malan, T, Jr. (2011). Regular exercise reverses sensory hypersensitivity in a rat neuropathic pain model: Role of endogenous opioids. Anesthesiology, 114, 940-948.

Stein, K. D., Goldman, M. S., \& Del Boca, F. K. (2000). The influence of alcohol expectancy priming and mood manipulation on subsequent alcohol consumption. Journal of Abnormal Psychology, 109, 106-115.

Testa, A., Nencini, P., \& Badiani, A. (2011). The role of setting in the oral self-administration of alcohol in the rat. Psychopharmacology, 215, 749-760

Tobler, P. N., Fiorillo, C. D., \& Schultz, W. (2005). Adaptive coding of reward value by dopamine neurons. Science, 307, 1642-1645. 
Wikler, A. (1959). Narcotics. In F. J. Braceland (Ed.), The effect of pharmacologic agents on the nervous system (pp. 334-355). Baltimore, MD, United States: Williams \& Wilkins.

Winger, G., Woods, J. H., Galuska, C. M., \& WadeGaluska, T. (2005). Behavioral perspectives on the neuroscience of drug addiction. Journal of the Experimental Analysis of Behavior, 84, 667-681.

Yahn, S. L., Watterson, L. R., \& Olive, M. F. (2013). Safety and efficacy of acamprosate for the treatment of alcohol dependence. Substance Abuse: Research and Treatment, 6, 1-12. doi:10.4137/SART.S9345

Yi, S. (2013). Heterogeneity of compulsive buyers based on impulsivity and compulsivity dimensions: A latent profile analytic approach. Psychiatry Research, 208, 174-182. 